\title{
BOND GRAPH MODEL OF A PIVOTING AXLE CONCEPT VEHICLE
}

\author{
Alex K. Beckerman \\ Donald Margolis \\ Jordan McCrone \\ Department of Mechanical and Aerospace Engineering \\ University of California, Davis \\ One Shields Avenue \\ Davis, CA, USA
}

\begin{abstract}
This paper presents a high-order vehicle model representative of the proposed automobile by Athena Technolo-G. Such a vehicle includes a novel pivoting axle concept that replaces the traditional steering and suspension mechanisms with axles and multi-axis bushings. This simplification dramatically reduces mechanical complexity with the intent of reducing developmental and manufacturing costs. The role of the steering and suspension mechanisms will be replicated using four independent in-wheel motors. The model put forth in this paper will aid in future studies to evaluate the efficacy of such a novel vehicle architecture. Specifically, this paper aims to capture the relevant dynamics between in-wheel motor torques and both handling and ride properties. Special attention is given to the multi-axis bushing that connects the pivoting axle and sprung mass. The sprung mass and both pivoting axles each have six degrees-of-freedom. A single aggressive maneuver is performed to showcase the model.
\end{abstract}

Keywords: Bond graph, vehicle, pivoting axle, in-wheel motor

\section{INTRODUCTION}

An automobile proposed by Athena Technolo-G, a concept of which is shown in Figure 1, replaces traditional steering and suspension mechanisms with an axle and multi-axis bushing at the front and rear of the vehicle. The role of the steering and suspension mechanisms will be replicated using four independent in-wheel motors. Removing the steering and suspension mechanisms will greatly reduce cost of design, fabrication, assembly, operation, and maintenance (Prous 2018). The pivoting axle technology is also expected to conserve energy as assembly plants are reduced in size as the fabrication, assembly, and storage needs are greatly simplified (Prous 2018). The proposed vehicle platform will likely consist of a structural frame built around an energy enclosure, two pivoting axle assemblies with their own electric motors, and a central controller (Prous 2018). It is thought that the pivoting axle technology may be applied to vehicles of all sizes, ranging from automobiles to large freight trailers, and be used in both manned and autonomous configurations (Prous 2018).

Such a vehicle relies on independent electric in-wheel motors at either end of each pivoting axle. Electric motors are capable of producing positive and negative torques at rates much faster and more predictable than traditional engine or brake torques. Additionally, it is only with independent torques that both longitudinal 
and yaw motion of the axle can be controlled. It is this motion of the pivoting axle that both propels and steers the vehicle. A central controller is required as even the simplest turning maneuvers would require coordination of all four electric motors. For this reason, and given the reduced mechanical complexity of this new platform, product development will change from a traditional hardware-centric philosophy to one of controls (Prous 2018).

As with any novel mechanical system, extensive modeling is required. This paper proposes a high fidelity vehicle model that aims to capture relevant handing and ride characteristics below $15 \mathrm{~Hz}$. With such a model, is it possible to study the wheel torque influence on vehicle yaw rate, vehicle body side-slip angle and rate, sprung mass roll angle and rate, vertical motion, and more. Consequently, it will be possible to obtain the motor performance required for sprung mass vertical motion control, sprung mass roll control, vehicle drive off control, and vehicle lateral dynamics control. Motor metrics of interest include maximum torque, speed, and actuation frequency. Additionally, such a model provides insight into bushing design, where the stiffness and damping properties along each axis can be understood in terms of performance trade-offs among the previously mentioned considerations.

The system is modeled using bond graphs, a graphical physical system modeling technique. A simple introduction to bond graphs is given in Section 2 while a thorough explanation of bond graphs is available in (Karnopp, Margolis, and Rosenberg 2012, Paynter 1961). Bond graphs are advantageous because they are graphical, modular, incorporate multiple energy domains, and provide system intuition. For these reasons, bond graphs are chosen over more conventional modeling techniques like Newton's or Lagrange methods.

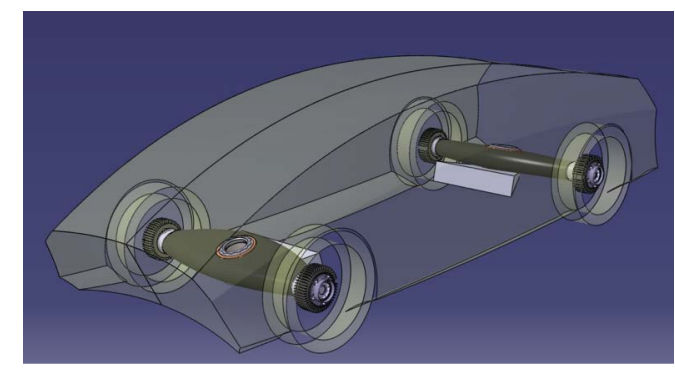

Figure 1: Vehicle concept drawing.

\section{BOND GRAPHS}

A bond graph is a collection of elements that, when combined, are used to describe physical systems. For example, elements exist that describe masses, springs, and dampers. Additional elements relate the forces and velocities associated with those elements. These elements are combined systematically to produce the final bond graph structure. A thorough explanation for each element is shown in (Karnopp, Margolis, and Rosenberg 2012, Paynter 1961).

When working with bond graphs, it is common to refer to forces and velocities as efforts and flows, respectively. This paper uses the terms interchangeably. Primarily, bond graph elements only provide relationships for efforts, $e$, flows, $f$, momenta, $p$, and displacements, $q$. Energy storage and dissipative elements are shown in Table 1 with their preferred causality, or their preferred input-output relationship. Non-causal relationships are not discussed in this paper. Energy storage elements (labeled as Inertia and Compliance in Table 1) are those that relate flows to a momenta or efforts to displacements. Dissipative elements (labeled as Resistance in Table 1) relate flows to efforts or vice-versa while removing energy from the system. Note that $\Phi$ represents a general linear or nonlinear function for each element. 
Table 1: Bond graph elements that either store or dissipate energy.

\begin{tabular}{|c|c|c|c|}
\hline Inertia & $I$ & $f=\Phi_{I}^{-1}(p)$ & Mass \\
\hline Compliance & $C$ & $e=\Phi_{C}^{-1}(q)$ & Spring \\
\hline Resistance & $R$ & $\begin{array}{c}e=\Phi_{R}(f) \\
f=\Phi_{R}^{-1}(e)\end{array}$ & Damper \\
\hline
\end{tabular}

Table 2: Various multiport bond graph elements.

\begin{tabular}{|c|c|c|}
\hline 1-Junction & $f_{i}=c$ & $\sum e_{i}=0$ \\
\hline 0-Junction & $\sum f_{i}=0$ & $e_{i}=c$ \\
\hline Transformer & $f_{2}=m f_{1}$ & $e_{1}=m e_{2}$ \\
\hline Gyrator & $f_{2}=r e_{1}$ & $e_{2}=r f_{1}$ \\
\hline
\end{tabular}

Various multiport elements are shown in Table 2. These elements relate efforts and flows while conserving power. A major advantage of bond graphs is the relationship between efforts and flows. Specifically, the efforts are constrained once the flows are known. Note that $m$ and $r$ are some value that relates the flows or efforts.

The final set of elements discussed are the effort and flow sources shown in Table 3. These elements do nothing more than prescribe either an effort or a flow as a function of time.

Table 3: Bond graph source elements.

\begin{tabular}{|c|c|c|c|}
\hline Effort source & $S_{e}$ & $e=e(t)$ & Force actuator \\
\hline Flow source & $S_{f}$ & $f=f(t)$ & Velocity source \\
\hline
\end{tabular}

The notation for the bonds themselves are shown in Figure 2 for sys 1 and sys 2 .The causality and direction of power flow is set by the vertical line and half arrow, respectively, at either end of the bond. The causal mark in this orientation states that sys 1 and sys 2 sets the flow and effort, respectively. A causal mark in the opposing orientation would signify that the opposite is true. Methods for determining causal and power flow orientation are provided in (Karnopp, Margolis, and Rosenberg 2012, Paynter 1961).

$$
\text { sys } 1 \stackrel{\text { effort }}{\stackrel{\text { flow }}{\longrightarrow}} \text { sys } 2
$$

Figure 2: A single bond with descriptions and power flow to the right.

\section{MODEL}

The sprung mass and axles each have six degrees-of-freedom: three translational and three rotational. They are connected by multi-axis bushings that are modeled using compliance and damping elements. These bushings are either pitched up or down relative to the sprung mass in a way that further couples modes, such as longitudinal and vertical or axle yaw and sprung mass roll. Tires at either end of each axle are free to rotate and have vertical stiffness. A torque representative of in-wheel motors is applied between each axle and tire, and a vertical velocity representative of the road profile is applied beneath the tire. Schematics are provided throughout this section while bond graphs are provided in Section 3.1.

Sprung mass top, side, and rear view schematics are shown in Figures 3, 4, and 5, respectively. $v_{s}$ and $\omega_{s}$ are the translational and rotational velocities of the sprung center of mass, and $v_{c 1}$ and $\omega_{c 1}$ are the translation and rotational velocities of the front bushing attachment point, respectively. The rear bushing is excluded for clarity. Subscripts $x, y$, and $z$ represent the longitudinal, lateral, and vertical directions, respectively, in a sprung mass fixed frame. Geometric parameters include the distance from the sprung center of mass to the front bushing attachment point, $a$, equivalent distance to the rear, $b$, sprung center of mass height, $h$, and front and rear rake angles, $\psi_{1}$ and $\psi_{2}$, respectively. Additional variables include the front and rear axle steer angle, $\delta_{1}$ and $\delta_{2}$, respectively. 

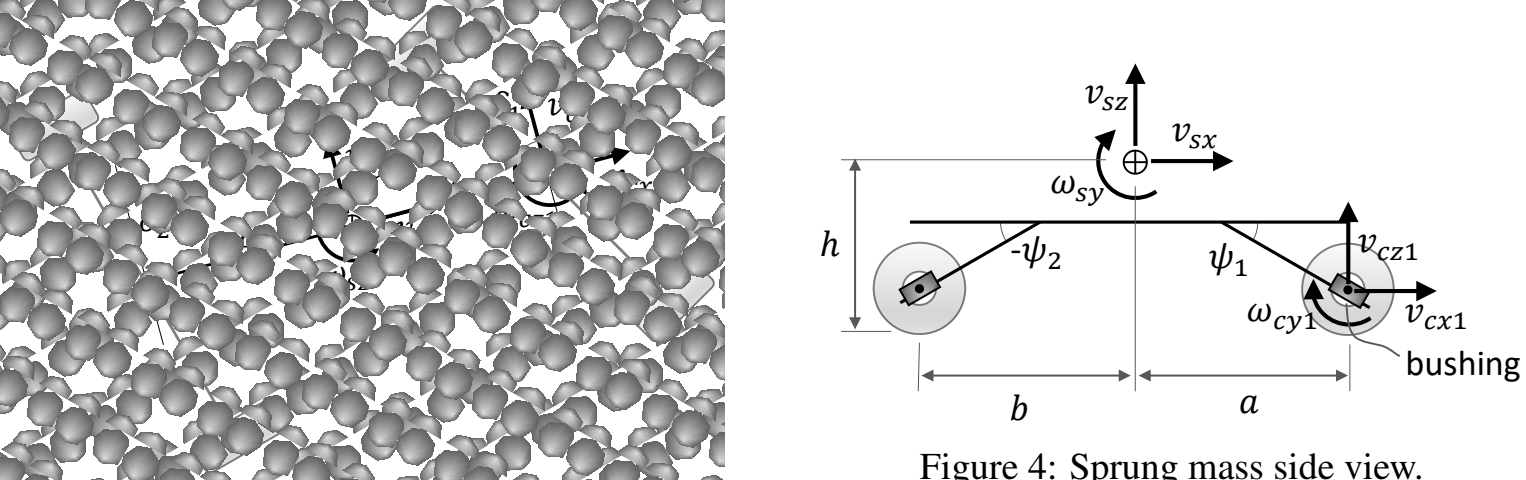

Figure 3: Sprung mass top view.

Figure 4: Sprung mass side view.

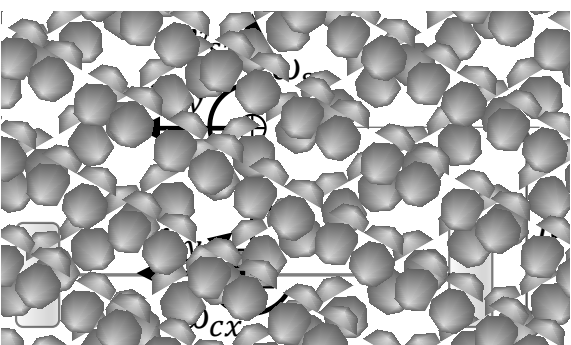

Figure 5: Sprung mass rear view.

Axle top, side, and rear view schematics are shown in Figures 6, 7, and 8, respectively, for either the front or rear axle. In future steps, each parameter or variable name is appended with the subscript 1 or 2 for the front or rear axle, respectively. $v_{a}$ and $\omega_{a}$ are the translational and rotational velocities of the axle center of mass, respectively. Subscripts $x, y$, and $z$ represent the longitudinal, lateral, and vertical directions, respectively, in an axle fixed frame. Additional velocities include the tire rolling velocity about the $y$ axis, $\omega_{t}$, wheel center longitudinal and vertical velocity, $v_{w c x}$ and $v_{w c z}$, respectively, and contact patch longitudinal and lateral velocity, $v_{c p x}$ and $v_{c p y}$, respectively. Subscript $l$ and $r$ indicate a left and right sided variable, respectively. Right sided variables are excluded for clarity. Geometry parameters include the tire radius, $R$, and the half-track width, $t$.
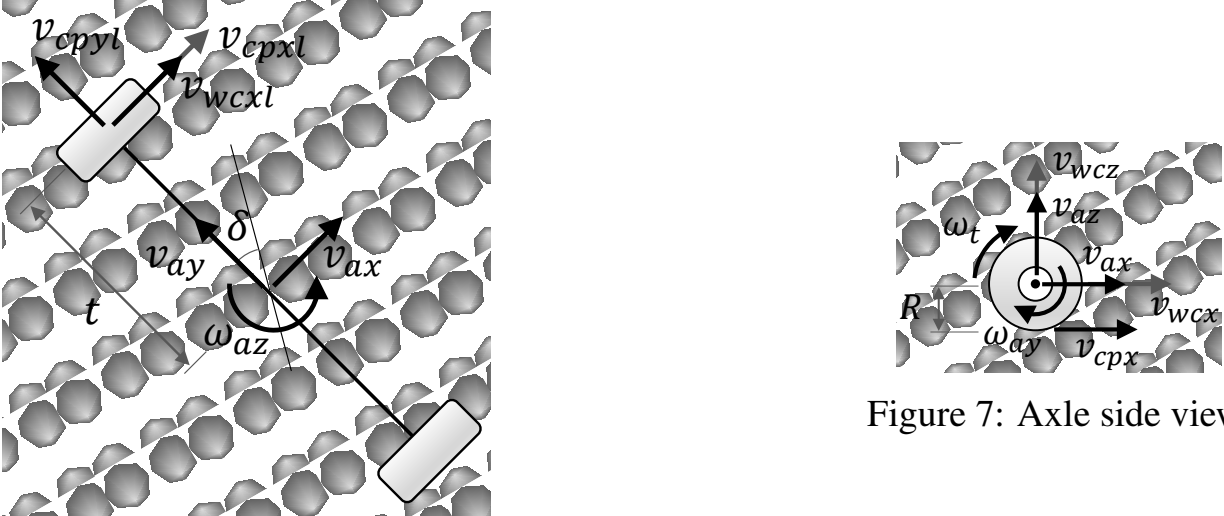

Figure 6: Axle top view.

Figure 7: Axle side view. 


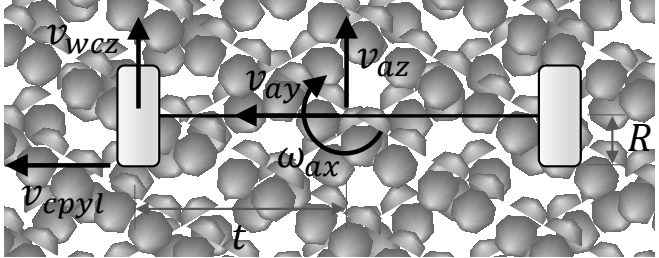

Figure 8: Axle rear view.

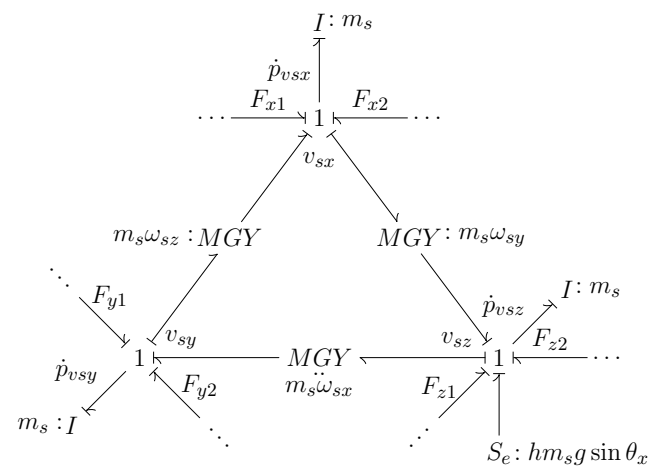

Figure 9: Translational sprung mass bond graph.

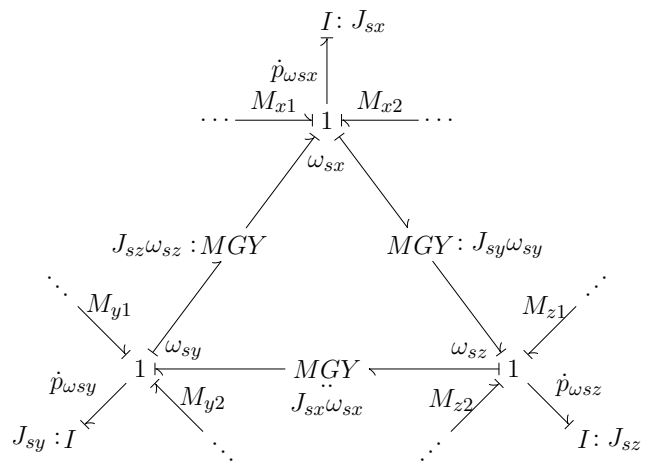

Figure 10: Translational sprung mass bond graph.

\subsection{Bond Graphs}

For clarity, the bond graph is divided into five modules: translational sprung mass, rotational sprung mass, sprung mass and bushing connection, bushing, and axle. There exists additional versions of the latter three modules for the rear of the vehicle. For clarity, these additional modules are discussed but not shown. Modules connect to one-another through bonds with ellipses.

The bond graph module for translational sprung mass elements is shown in Figure 9. Included are the translational sprung mass velocities and associated bonds. The general structure, including the modulated gyrators arranged as shown, are used to represent the dynamics of a body fixed rotating coordinate system. Details of this bond graph structure are shown in (Karnopp, Margolis, and Rosenberg 2012). New parameters and variables are introduced that are in addition to those discussed in Section 3. They include the sprung mass, $m_{s}$, acceleration due to gravity, $g$, and roll angle, $\theta_{x}$. Also shown are input forces from other bond graph modules, $F_{x}, F_{y}$, and $F_{z}$, where additional subscripts 1 and 2 indicate front and rear vehicle components, respectively. Finally, there exists momentum variables, $p$, where each subscript corresponds with the associated sprung mass translational velocity.

The bond graph module for rotational sprung mass elements is shown in Figure 10. As with the translational sprung mass bond graph, this new bond graph structure is a result of the body fixed rotating coordinate system. $J_{s}$ is the sprung mass polar moment of inertia about the $x, y$, or $z$ axis, indicated by additional subscripts. Also shown are input moments from other bond graph modules, $M_{x}, M_{y}$, and $M_{z}$, where additional subscripts 1 and 2 indicate front and rear vehicle components, respectively. Finally, there exists momentum variables, $p$, where each subscript corresponds with the associated sprung mass rotational velocity.

The bond graph module that connects the sprung mass to the front bushing is shown in Figure 11. For clarity, the bond graph for the rear axle is excluded. This bond graph contains both sprung mass and front bushing translational and rotational velocities and the kinematics that relates them. These kinematic velocity relationships are found by inspection and are shown in Equations 1 and 2 for the front and rear bushings, 
respectively. Input forces and moments, $F_{c}$ and $M_{c}$, respectively, with additional subscripts $x, y$, and $z$ represent intermediate forces that are calculated within a separate bond graph module. All other parameters and variables were previously defined in Section 3.

$$
\begin{gathered}
v_{c x 2}=v_{s x}-a \omega_{s y} . \\
v_{c y 2}=v_{s y}+h \omega_{s x}-b \omega_{s z} . \\
v_{c z 2}=v_{s z}+b \omega_{s y} .
\end{gathered}
$$

$$
\begin{gathered}
v_{c x 2}=v_{s x}-a \omega_{s y} . \\
v_{c y 2}=v_{s y}+h \omega_{s x}-b \omega_{s z} . \\
v_{c z 2}=v_{s z}+b \omega_{s y} .
\end{gathered}
$$

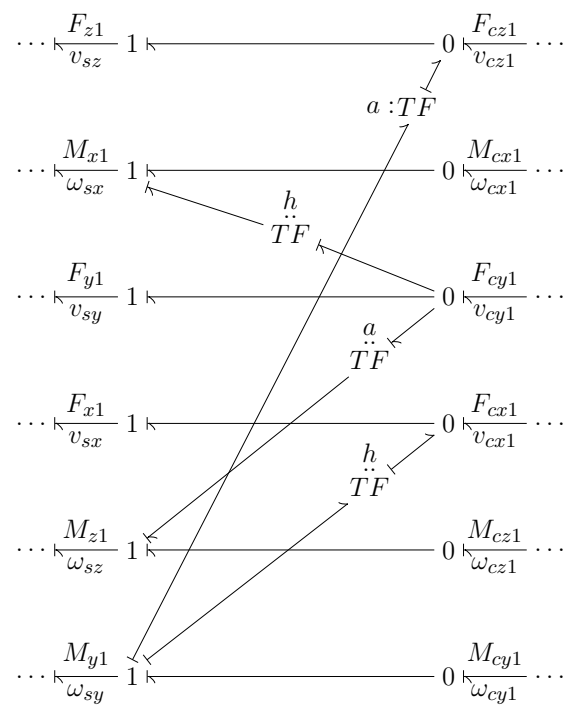

Figure 11: Sprung mass and bushing relationship bond graph for front of vehicle.

The bond graph module that contains the bushing stiffness and damping elements is shown in Figure 12. This bond graph is equivalent for both front and rear bushings. Therefore, subscripts 1 and 2 are not required. $q_{b}, k_{b}$ and $b_{b}$ are bushing deflection, stiffness, and damping coefficients, where additional subscripts $x, y$ and $z$ indicate the axis, and additional subscript $\tau$ indicates rotation. The force or moments within the bushings are given by Equation 3, where the above subscripts are excluded for clarity.

$$
\begin{gathered}
F_{b}=k_{b} q_{b}+b_{b} \dot{q}_{b} . \\
M_{b}=k_{\tau b} q_{\tau b}+b_{\tau b} \dot{q}_{\tau b} .
\end{gathered}
$$

Bushing relative velocities are defined in a bushing fixed frame that has been rotated from a sprung mass fixed frame through an angle $\psi$ about the $y$-axis. As such, relative bushing velocities in the bushing fixed frame are related to those in the sprung mass fixed frame, shown with bars above the variable, using the transformation shown in Equation 4. Note that $c \psi$ and $s \psi$ represent the cosine and sine of the anle $\psi$. This notation will be used throughout the remainder of this paper for general angles. The same transformation applies to rotational velocities and can be obtained by appending the subscript $\tau$ to Equation 4 .

$$
\begin{gathered}
\dot{q}_{b x}=c \psi \dot{\bar{q}}_{b x}+s \psi \dot{\bar{q}}_{b z} . \\
\dot{q}_{b z}=-s \psi \dot{\bar{q}}_{b x}+c \psi \dot{\bar{q}}_{b z} .
\end{gathered}
$$


Additionally, axle longitudinal and lateral velocities, $v_{a x}$ and $v_{a y}$, respectively, are defined in an axle fixed frame and must first be rotated into a sprung mass fixed frame. These rotated velocities, $\bar{v}_{a x}$ and $\bar{v}_{a y}$, are required for calculation of the bushing relative velocities and shown in Equation 5. The rotation is about the $z$-axis by the steer angle, $\delta$.

$$
\begin{aligned}
& \bar{v}_{a x}=c \delta v_{a x}-s \delta v_{a y} . \\
& \bar{v}_{a y}=s \delta v_{a x}+c \delta v_{a y} .
\end{aligned}
$$

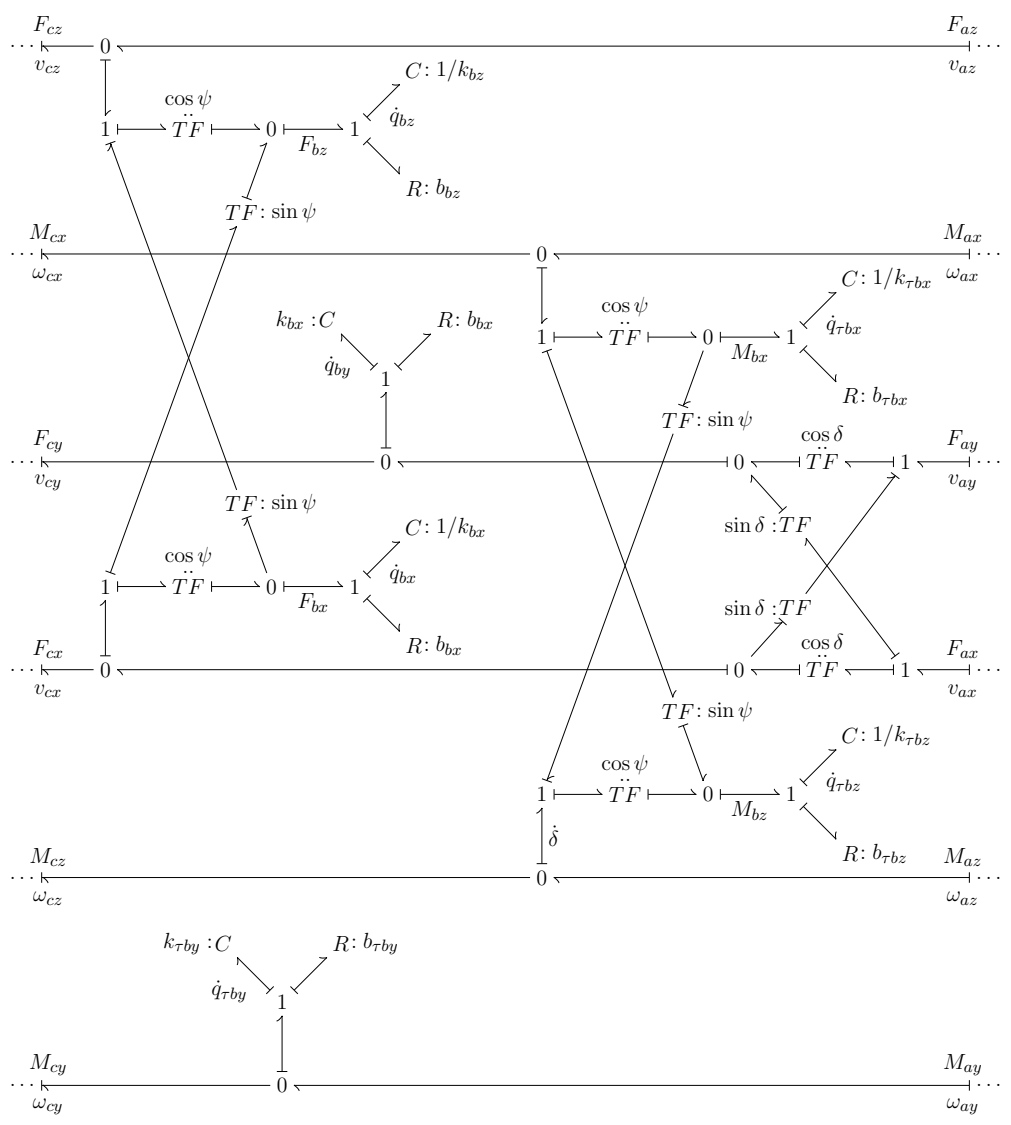

Figure 12: Bushing bond graph.

The bond graph module containing all axle elements is shown in Figure 13. The axle has six degrees-offreedom such that there exists three translational and three rotational velocities. These velocities are shown as $v_{a}$ and $\omega_{a}$ with subscripts $x, y$, and $z$ to indicate the axis. Momentum variables, $p$, are shown with subscripts that correspond to the associated velocity. Each velocity also has an associated inertial element: $m_{a}, J_{a x}, J_{a y}$, and $J_{a z}$. Input forces and moments, $F_{a}$ and $M_{a}$, respectively, with additional subscripts $x, y$, and $z$ represent intermediate forces that are calculated within a separate bond graph module. The vertical tire relative velocity, $\dot{q}_{t}$, is seen in the bond graph as the difference between the vertical road input velocity, $v_{i}$, and the vertical wheel center velocity, $v_{w c z}$, where $v_{w c z}$ is evaluated using Equation 6. Additionally, the tire has stiffness $k_{t}$ that acts on this relative velocity, resulting in a vertical tire fore, $F_{z}$. Subscripts $l$ and $r$ indicate the left or right side of the vehicle. 


$$
\begin{aligned}
& v_{w c z l}=v_{a z}+t \omega_{a x} . \\
& v_{w c z r}=v_{a z}-t \omega_{a x} .
\end{aligned}
$$

Longitudinal and lateral tire forces, $F_{x}$ and $F_{y}$, respectively, act at the contact patch longitudinal and lateral velocities, $v_{c p x}$ and $v_{c p y}$, respectively. $v_{c p x}$ and $v_{c p y}$ are obtained from inspection and evaluated using Equation 7. They shown to be a function of wheel center longitudinal velocity, $v_{w c x}$, which is also obtained from inspection and evaluated using Equation 8. Subscripts $l$ and $r$ indicate the left or right side of the vehicle.

$$
\begin{gathered}
v_{c p x}=v_{w c x}-R \omega_{u s} . \\
v_{c p y}=v_{a y}+R \omega_{a x} .
\end{gathered}
$$

$$
\begin{aligned}
& v_{w c x l}=v_{a x}-t \omega_{a z} . \\
& v_{w c x r}=v_{a x}+t \omega_{a z} .
\end{aligned}
$$

Finally, tire torques, $\tau_{i}$, are applied to the tire rolling angular velocity, $\omega_{t}$.

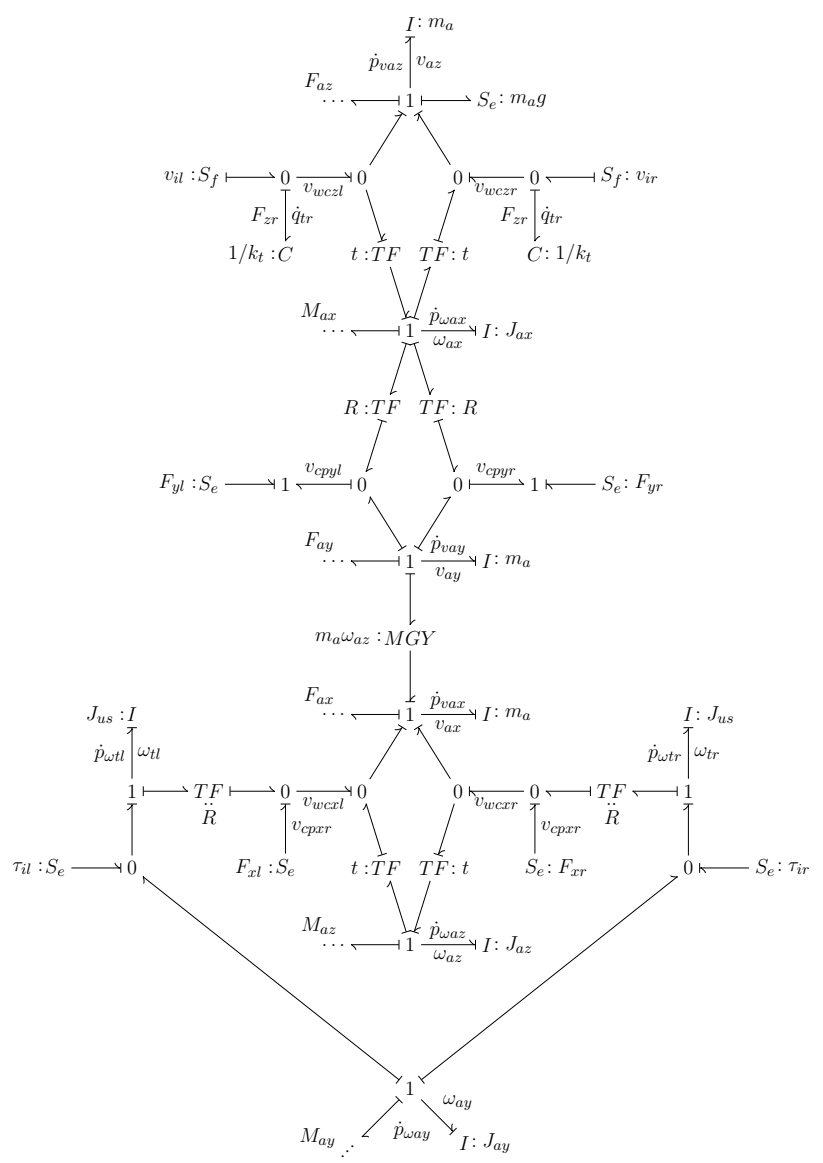

Figure 13: Axle bond graph. 


\subsection{Tires}

Longitudinal and lateral tire forces, $F_{x}$ and $F_{y}$, respectively, are related to the slip ratio, $k$, slip angle, $\alpha$, and tire normal force, $F_{z}$, using the well known Pacejka model (Pacejka 2005). $k$ is the relative longitudinal velocity between the contact patch and the road and normalized by some value, and $\alpha$ is the relative angle between the direction of travel and the centerline of the tire. These three variables, $\alpha, k$, and $F_{z}$, are evaluated using Equation 9, where $R, k_{t}$, and $q_{t}$ are the tire radius, tire vertical stiffness, and tire vertical deflection, respectively, and $\omega_{t}, v_{w c x}$, and $v_{c p y}$ were discussed in Section 3.1.

$$
\begin{gathered}
k=\frac{R \omega_{t}-v_{w c x}}{\max \left(R \omega_{t}, v_{w c x}\right)} . \\
\alpha=-\tan ^{-1}\left(\frac{v_{c p y}}{v_{w c x}}\right) . \\
F_{z}=k_{t} q_{t} .
\end{gathered}
$$

In pure slip conditions, longitudinal and lateral tire force are plotted against slip ratio and angle, respectively, where all other variables are assumed constant. This is shown for longitudinal tire force in Figure 14. The effect of coupling between slip ratio and angle, and similarly longitudinal and lateral tire force, result in a decrease in both forces. Increasing normal force, however, increases the maximum obtainable net force (evaluated as the Euclidean norm of the perpendicular forces) until saturation is reached. Details regarding slip ratio, slip angle, and normal force coupling are found in (Pacejka 2005).

\subsection{Equations}

Equations are obtained using standard bond graph techniques. They are not presented in order and would require sorting to run in simulation. Additional intermediate equations (those not presented throughout the paper) are presented in Equations 10 - 13 and equations of motion are presented in Equation 14 - 17.

Momenta are converted to velocities in Equation 10, where subscripts $x, y$, and $z$ (for each equation) and 1 and 2 (for axle only) are excluded for clarity. Forces applied to the axle are shown in Equation 11, where subscripts 1 and 2 are excluded for clarity. Forces applied to the sprung mass that originate from the front and rear axle are shown in Equations 12 and 13, respectively.

$$
\begin{gathered}
v_{s}=p_{v s} / m_{s}, \quad \omega_{s}=p_{\omega s} / J_{s} . \\
v_{s}=p_{v a} / m_{a}, \quad \omega_{a}=p_{\omega a} / J_{a} . \\
\omega_{t}=p_{\omega t} / J_{u s} . \\
F_{a x}=c \delta F_{x}+s \delta F_{b y} . \\
F_{a y}=c \delta F_{b y}-s \delta F_{x} . \\
F_{a z}=c \psi F_{b z}-s \psi F_{b x} . \\
M_{a x}=c \psi M_{b x}+s \psi M_{b z} . \\
M_{a y}=M_{b y} . \\
M_{a z}=c \psi M_{b z}-s \psi M_{b x} .
\end{gathered}
$$




$$
\begin{gathered}
F_{x 1}=c \psi_{1} F_{b x 1}+s \psi_{1} F_{b z 1} . \\
F_{y 1}=F_{b y 1} . \\
F_{z 1}=c \psi_{1} F_{b z 1}-s \psi_{1} F_{b x 1} . \\
M_{x 1}=c \psi_{1} M_{b x 1}+s \psi_{1} M_{b z 1}+h F_{b y 1} . \\
M_{y 1}=M_{b y 1}-h F_{x 1}-a F_{z 1} . \\
M_{z 1}=c \psi_{1} M_{b z 1}-s \psi_{1} M_{b x 1}+a F_{b y 1} .
\end{gathered}
$$

$$
\begin{gathered}
F_{x 2}=c \psi_{2} F_{b x 2}+s \psi_{2} F_{b z 2} . \\
F_{y 2}=F_{b y 2} . \\
F_{z 2}=c \psi_{2} F_{b z 2}-s \psi_{2} F_{b x 2} . \\
M_{x 2}=c \psi_{2} M_{b x 2}+s \psi_{2} M_{b z 2}+h F_{b y 2} . \\
M_{y 2}=M_{b y 2}-h F_{x 2}+b F_{z 2} . \\
M_{z 2}=c \psi_{2} M_{b z 2}-s \psi_{2} M_{b x 2}-b F_{b y 2} .
\end{gathered}
$$

Sprung mass equations of motion are shown in Equation 14. Axle equations of motion are shown in Equation 15 - 16, where subscripts 1 and 2 are removed for clarity. Additional equations of motion include front and real steer angle, $\delta_{1}$ and $\delta_{2}$, respectively, and roll angle, $\theta_{x}$. These are shown in Equation 17.

$$
\begin{aligned}
& \dot{p}_{v s x}=F_{x 1}+F_{x 2}+m_{s} \omega_{s z} v_{s y}-m_{s} \omega_{s y} v_{s z} . \\
& \dot{p}_{v s y}=F_{y 1}+F_{y 2}+m_{s} \omega_{s x} v_{s z}-m_{s} \omega_{s z} v_{s x} . \\
& \dot{p}_{v s z}=F_{z 1}+F_{z 2}-m_{s} g+m_{s} \omega_{s y} v_{s x}-m_{s} \omega_{s x} v_{s y} \text {. } \\
& \dot{p}_{\omega s x}=M_{x 1}+M_{x 2}+h m_{s} g \sin \theta_{x}+\ldots \\
& \ldots+\left(J_{s y}-J_{s z}\right) \omega_{s y} \omega_{s z} \text {. } \\
& \dot{p}_{\omega s y}=M_{y 1}+M_{y 2}+\left(J_{s z}-J_{s x}\right) \omega_{s z} \omega_{s x} . \\
& \dot{p}_{\omega s z}=M_{z 1}+M_{z 2}+\left(J_{s x}-J_{s y}\right) \omega_{s x} \omega_{s y} . \\
& \dot{p}_{v a x}=-F_{a x}+F_{x l}+F_{x r}+m_{a} \omega_{a z} v_{a y} . \\
& \dot{q}_{b x}=c \psi\left(c \delta v_{a x}-s \delta v_{a y}-v_{c x}\right)-s \psi\left(v_{a z}-v_{c z}\right) . \\
& \dot{p}_{v a y}=-F_{a y}+F_{y l}+F_{y r}-m_{a} \omega_{a z} v_{a x} \text {. } \\
& \dot{p}_{v a z}=-F_{a z}+F_{z l}+F_{z r}-m_{a} g \text {. } \\
& \dot{p}_{\omega a x}=-M_{a x}+t\left(F_{z l}-F_{z r}\right)+R\left(F_{y l}+F_{y r}\right) . \\
& \dot{p}_{\omega a y}=-M_{a y}-\tau_{i l}-\tau_{i r} . \\
& \dot{p}_{\omega a z}=-M_{a z}-t\left(F_{x l}-F_{x r}\right) \text {. } \\
& \dot{p}_{\omega t l}=\tau_{i l}-R F_{x l} . \\
& \dot{p}_{\omega t r}=\tau_{i r}-R F_{x r} \text {. } \\
& \dot{q}_{b y}=c \delta v_{a y}+s \delta v_{a x}-v_{c y} . \\
& \dot{q}_{b z}=c \psi\left(v_{a z}-v_{c z}\right)+s \psi\left(c \delta v_{a x}-s \delta v_{a y}-v_{c x}\right) \text {. } \\
& \dot{q}_{\tau b x}=c \psi\left(\omega_{a x}-\omega_{c x}\right)-s \psi\left(\omega_{a z}-\omega_{c z}\right) . \\
& \dot{q}_{\tau b y}=\omega_{a y}-\bar{\omega}_{a y} . \\
& \dot{q}_{\tau b z}=c \psi\left(\omega_{a z}-\omega_{c z}\right)+s \psi\left(\omega_{a x}-\omega_{c x}\right) . \\
& \dot{q}_{t l}=v_{i l}-v_{w c z l} \text {. } \\
& \dot{q}_{t l}=v_{i r}-v_{w c z r} \text {. } \\
& \dot{\delta}_{1}=\omega_{a z 1}-\omega_{s z} . \\
& \dot{\delta}_{2}=\omega_{a z 2}-\omega_{s z} \text {. } \\
& \dot{\theta}_{x}=\omega_{s x} \text {. }
\end{aligned}
$$




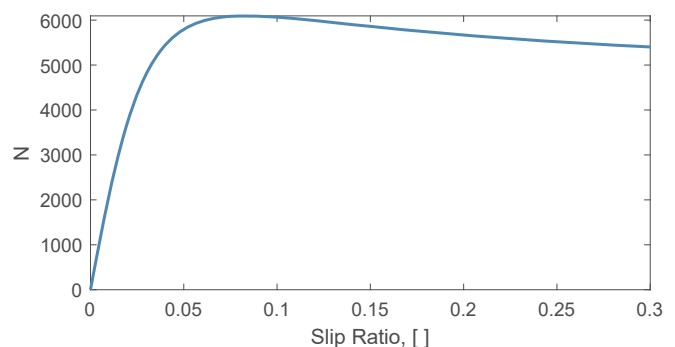

Figure 14: Longitudinal tire force versus slip ratio.

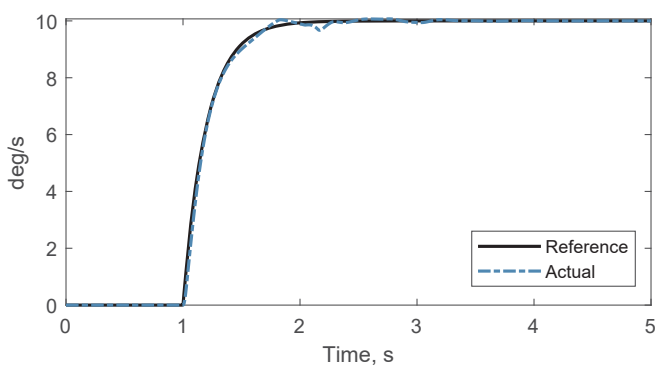

Figure 16: Yaw rate.

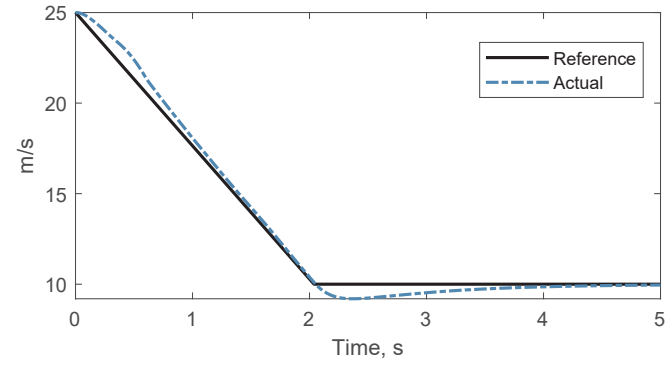

Figure 15: Longitudinal velocity.

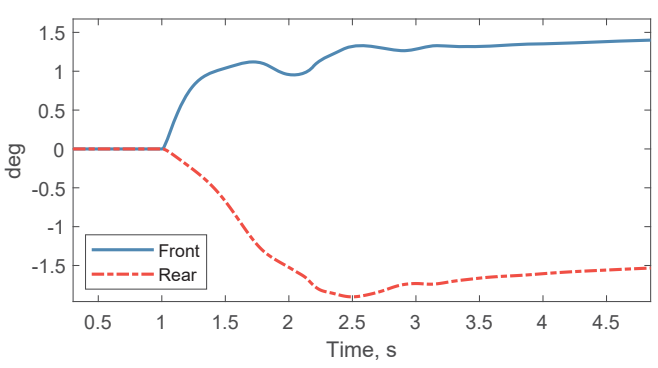

Figure 17: Axle steer angle.

\section{RESULTS}

A single test maneuver is performed: a sudden deceleration followed by a sudden turn. The vehicle is commanded to decelerate at $0.75 \mathrm{~g}$ 's from $25 \mathrm{~m} / \mathrm{s}(56 \mathrm{MPH})$ to $10 \mathrm{~m} / \mathrm{s}(22 \mathrm{MPH})$. Approximately one second into this maneuver, the vehicle is then commanded to following a reference yaw rate from 0 to 10 $\mathrm{deg} / \mathrm{s}$. These velocity and yaw rate commands are converted to tire torque inputs using a novel feedback control algorithm. The details of this algorithm are not discussed in this paper. This maneuver is aggressive and results in highly nonlinear tire behavior, particularly due to normal force variations that result from load transfer.

Longitudinal velocity and yaw rate responses are shown in Figures 15 and 16, respectively, and compared to their reference commands discussed above. It is clear that the vehicle is capable of following the target with errors likely considered acceptable to any engineer in the automotive community. Additionally, it is likely that future control development will result in improved performance. The front and rear steer angles are shown in Figure 17, where this particular control algorithm makes use of a larger rear steer angle to assist in the turn. Each tire torque is shown in Figure 18, where anything below the $-500 \mathrm{Nm}$ line is accomplished with mechanical brakes and anything above the $-500 \mathrm{Nm}$ line is accomplished with the electric motor. -500 $\mathrm{Nm}$ is chosen as it is a reasonable torque saturation limit for a standard electric vehicle in-wheel motor. Given the ability to produce negative torques down to $-500 \mathrm{Nm}$, it is therefore possible to recuperate energy through regenerative braking. Both power and energy (assumed to start at zero KWh) are shown in Figure 19 , where positive power is defined as energy absorbed.

\section{CONCLUSIONS}

This paper presented a high-order vehicle model representative of the proposed automobile by Athena Technolo-G, where traditional steering and suspension elements are replaced with multi-axis bushings and axles at the front and rear of the vehicle. The model was comprised of a sprung mass with two axles, each with six degrees of freedom, and separated by a series of springs and dampers. The springs and dampers were angled in a way that provided realistic coupling between dynamic modes. Tires at both ends of each 


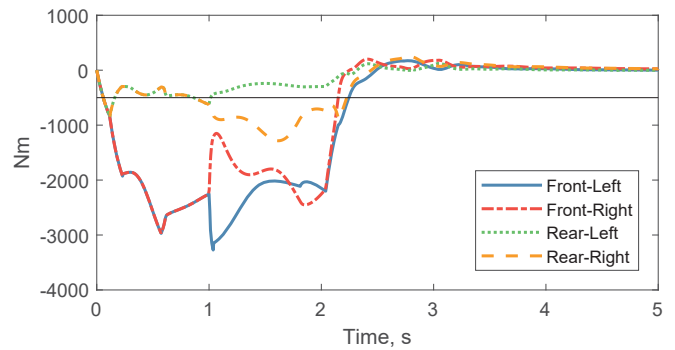

Figure 18: Tire input torques.

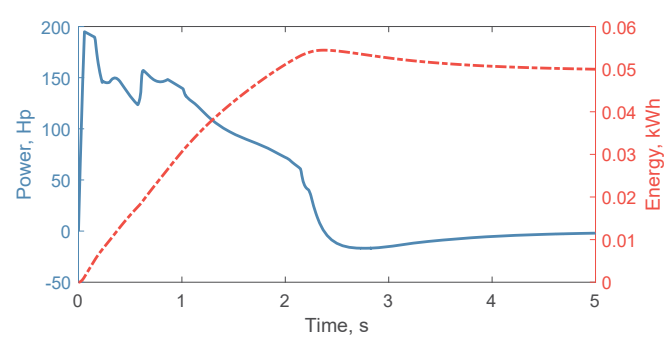

Figure 19: Electric motor power and energy.

axle had a vertical stiffness and were free to rotate. Torques between tires and axles were used to represent torques that may be applied by in-wheel electric motors. A novel control algorithm was used to control these torques to illustrate the model in a single aggressive test maneuver. Given the successful tracking of a realistic test maneuver such as this helps validate that this novel pivoting axle architecture may be suitable for passenger vehicle applications.

Future studies should be conducted to better understand the relationship between vehicle parameters and performance, including both dynamic capabilities and motor requirements. Additionally, future studies should go beyond simple longitudinal velocity and yaw rate control. This may include inertial position, lateral acceleration, and body slip angle control. Vehicle ride must also be addressed as stiff rubber bushings and no standard suspension are likely to have a negative impact. It may be possible to address this using control of the in-wheel motors depending on bandwidth limitations. Any novel vehicle requires extensive trial and error, something that can be assisted through future simulation of models such as this.

\section{REFERENCES}

Karnopp, D. C., D. L. Margolis, and R. C. Rosenberg. 2012. System dynamics: modeling, simulation, and control of mechatronic systems. John Wiley \& Sons.

Pacejka, H. 2005. Tire and vehicle dynamics. Elsevier.

Paynter, H. M. 1961. Analysis and Design of Engineering Systems; Class Notes for M.I.T. Course 2,751. Cambridge, MA, M.I.T.

Prous, M. 2018, 11. Automotive - Most Efficient. Athena Technolo-G. https://www.athenatechno.tech/automobile.

\section{AUTHOR BIOGRAPHIES}

ALEX K. BECKERMAN is a mechanical engineer in the Hyundai Center of Excellence in Vehicle Dynamic Systems \& Control at the University of California, Davis. His specialty is in the field of dynamic systems and controls as applied to automotive systems. His email is akbecker@ucdavis.edu.

DONALD L. MARGOLIS is professor emeritus at the University of California, Davis. His teaching and research is in the broad areas of modeling, simulation, and control of physical systems with particular emphasis on vehicle dynamics, control, and vibrations. His email is dlmargolis@ ucdavis.edu.

D. JORDAN MCCRONE is a graduate student researcher in the Hyundai Center for Excellence at UC Davis, a collaborative venture between students and faculty at UC Davis and visiting engineers from Hyundai in Korea. His research interest is in vehicle dynamics with a particular focus on control systems and advanced modeling techniques for multi-energy domain systems. His email is djmccrone@ucdavis.edu. 